\title{
Viscous Oil Occurrences, Biodegradation and Tar Mats. Advances and Knowledge Gaps
}

\section{Steve Larter, Schlumberger}

Heavy and viscous oil occurrences can be produced by a variety of in-reservoir mechanisms, including biodegradation of light oils, primary sourcing of heavy oil from type II-S kerogen rich source rocks, and by the segregation mechanisms related to oilfield charging and tectonic history that result in the formation of tar mats. Advances in the understanding of heavy oil origin through biodegradation, the dominant process of heavy oil formation, have been dramatic in the last decade, with major biogeochemical and petroleum geochemical discoveries. Anaerobic biological conversion of liquid petroleum hydrocarbons to methane in oil reservoirs (methanogenesis), on geological timescales, occurs through biological action of syntrophic bacteria and methanogenic archaea at temperatures as low as $15^{\circ} \mathrm{C}$. Earlier geochemical predictions that the microorganisms that biodegrade crude oil to produce heavy oil exist predominantly in the transition zone of oil fields has recently been recently verified by combined microbiological and petroleum geochemical approaches (Bennett et al, 2013). The bottom bioreactor configuration of biodegradation provides an ideal setting to produce compositional and fluid property gradients that are very sensitive to barrier and baffle occurrence within the oil column. This provides an ideal geological environment for barrier and baffle detection and assessment.

The origin of tar mats remains much more equivocal, although it is clear that biodegradation does not produce classical tar mats characterized by sharp compositional steps with their hosting lighter oil columns. While there have been new approaches to understanding the impact of asphaltene disposition in crude oils on compositional gradients in light oil columns, the interrelationships between oil column, tar mat and intra reservoir barriers and baffles remain poorly described and there have been no comprehensive high resolution petroleum geochemical studies of tar mat occurrences in light oil fields since the late $20^{\text {th }}$ century. In this paper, we review the detailed molecular studies of tar mat occurrence reported in the literature and identify the petroleum geochemical knowledge gaps and methodology gaps that exist, if the mechanistic origins of tar mats in individual settings are to be fully ascertained. Specifically, we examine recent advances in organic petrographic methods, especially microFTIR methods, that can provide information about small scale chemical variability in viscous oil occurrences and help understand the origin and timing of the emplacement of tar mats. 